\title{
The effects of cyproheptadine on locomotion and on spasticity in patients with spinal cord injuries
}

\author{
$M$ Wainberg, H Barbeau, S Gauthier
}

\begin{abstract}
The effects of cyproheptadine, a serotonergic antagonist, were studied in seven patients with spastic paresis of spinal origin. Six patients were included in a double blind crossover trial (maximal dose $24 \mathrm{mg} /$ day). The patients were evaluated on both their spasticity and locomotor function. Four of the patients also participated in an open trial in which cyproheptadine was administered for a minimum of six months at optimal dose. Patients walked on a treadmill at full weight bearing when possible, or with $40 \%$ of their body weight externally supported, as required, by an overhead harness system. Cyproheptadine considerably decreased the sustained ankle clonus and episodes of spontaneous spasms observed in all the patients who previously presented these manifestations of spasticity. Two patients who required body weight support (BWS) during locomotion could walk at full weight bearing during cyproheptadine therapy. A more normal timing of EMG patterns in these patients during cyproheptadine therapy was associated with temporal distance changes and marked improvement of joint angular displacement. In contrast, the other patients showed marginal changes in the EMG and the kinematic pattern but eventually managed to walk at a higher speed. These preliminary results suggest that cyproheptadine can reduce spasticity and enhance locomotor function in spinal cord injured patients.
\end{abstract}

School of Physical and Occupational Therapy, McGill University, Montreal, Canada

M Wainberg

H Barbeau

$S$ Gauthier *

Present address ${ }^{\star}$.

McGill Centre for Studies on

Aging, Montreal General

Hospital, 1650 Cedar

Avenue, Montreal, Quebec

H3G 1 A4 Canada.

Correspondence to:

H Barbeau, School of

Physical and Occupational

Therapy, McGill University

3654 Drummond Street,

Montreal, Quebec H3G 1 Y5

Canada.

Received 17 May 1989

and in revised form

27 November 1989

Accepted 6 December 1989
Serotonin (5-hydroxytryptamine or 5-HT) has been implicated in many physiological functions, including inhibition of spinal afferent processing, ${ }^{1}$ and spinal neuronal activity. ${ }^{2-4}$ The majority of the serotonin in the spinal cord was found to originate from the raphe nuclei in the medulla $a^{5}$ and project to the ventral, dorsal and lateral horns of the spinal cord. ${ }^{6}$ Of particular interest to this study is the important role attributed to the 5HT system in modulating the spasticity-like behaviour and the locomotor pattern in chronic spinal animals, in which all the descending fibres, including the bulbo-spinal serotonergic terminals below the transection, have disappeared. Administering serotonergic agonists, which act on 5-HT receptors below the transection, results in a marked increase in spontaneous electromyographic (EMG) activity, and an increased manifestation of spastic features such as hyperactive nociceptive and tendon reflexes, as well as increased resistance to passive movement, spasms, and clonus. $^{7-9}$ Cyproheptadine, a serotonergic antagonist, has been shown in the chronic spinal animal, to partially or completely antagonise these spastic features. ${ }^{7-9}$ In chronic spinal cats, injection of 5-HT agonists during a well established locomotor pattern resulted in a marked increase in EMG amplitude in both axial and limb muscles, and a marked increase in spinal reflexes. Cyproheptadine largely reverses the effects of 5-HT agonists on the locomotor pattern and spinal reflexes. ${ }^{1011}$

In humans, the serotonergic descending system may also play an important role in modifying the excitability of spinal neurons and in modulating motor acts such as locomotion. In an earlier study, Barbeau and collaborators $^{12}$ presented evidence to suggest that cyproheptadine reduces clonus and muscle spasms in patients with spinal cord injuries (SCI) while producing only a mild decrease in dynamic muscle strength. These promising findings led to a pilot case study of the effects of cyproheptadine on the modulation of locomotor function in two spastic SCI patients. ${ }^{613}$ In this investigation, we extend the study of the effects of cyproheptadine to a larger patient population with variable degrees of spasticity-related symptoms and locomotor function.

\section{Method}

Subjects

The study included eight patients with spastic paresis of spinal origin (table 1). The patients included were those presenting with spontaneous episodes of involuntary movements (spasms, clonus), able to demonstrate active movement of the lower extremities, and capable of transferring and standing with or without walking aids. The patients could be classified into two groups, based on their functional status (table 1). Patients in group A were wheelchair-bound due to severe spasticity $\left(\mathbf{P}_{1}, \mathbf{P}_{2}\right)$. Patients in group $\mathbf{B}$ could walk overground with aids $\left(P_{3}, P_{4}, P_{5}, P_{6}, P_{7}\right.$ and $\left.P_{8}\right)$. Informed consent was obtained in all cases.

Study design

Six subjects $\left(P_{1}, P_{2}, P_{4}, P_{6}, P_{7}\right.$ and $\left.P_{8}\right)$ were included in a double blind crossover trial (short term study). $P_{3}$ participated only in a long term 


\begin{tabular}{|c|c|c|c|c|c|c|c|c|}
\hline \multirow[b]{2}{*}{ Subject } & \multirow[b]{2}{*}{ Sex } & \multirow[b]{2}{*}{ Age } & \multirow[b]{2}{*}{ Diagnosis } & \multirow{2}{*}{$\begin{array}{l}\text { Time after } \\
\text { the lesion }\end{array}$} & \multirow{2}{*}{$\begin{array}{l}\text { Functional } \\
\text { status }\end{array}$} & \multirow[b]{2}{*}{ Clonus } & \multicolumn{2}{|l|}{ Dosage } \\
\hline & & & & & & & Short & Long \\
\hline $\begin{array}{l}P_{1} \\
P_{2} \\
P_{3} \\
P_{4} \\
P_{5} \\
P_{6}\end{array}$ & $\begin{array}{l}\mathbf{M} \\
\mathbf{M} \\
\mathbf{M} \\
\mathbf{M} \\
\mathbf{M} \\
\mathbf{M}\end{array}$ & $\begin{array}{l}24 \\
24 \\
56 \\
56 \\
23 \\
31\end{array}$ & $\begin{array}{l}\mathrm{C}-8 \\
\mathrm{~T}-4 \\
\mathrm{SP} \\
\mathrm{T}-11 \\
\mathrm{C}-4 \\
\mathrm{C}-6\end{array}$ & $\begin{array}{c}1 \\
1 \\
7 \\
1 \\
>1 \\
15\end{array}$ & $\begin{array}{l}\text { wheelchair } \\
\text { wheelchair } \\
1 \text { cane } \\
2 \text { canes } \\
1 \text { cane } \\
2 \text { elbow }\end{array}$ & $\begin{array}{l}S \\
S \\
S \\
S\end{array}$ & $\begin{array}{l}24 \\
24 \\
\text { NA } \\
24 \\
\text { Withdrew } \\
24\end{array}$ & $\begin{array}{l}\text { NA } \\
24 \\
24 \\
\text { NA } \\
\\
24\end{array}$ \\
\hline$P_{7}$ & $\mathbf{F}$ & 24 & C-6 & 4 & $\begin{array}{l}\text { crutches } \\
2 \text { elbow } \\
\text { crutches }\end{array}$ & $\mathbf{S}$ & 24 & 12 \\
\hline $\mathbf{P}_{\mathrm{k}}$ & $\mathbf{M}$ & 27 & $C-4-5$ & 4 & 1 cane & - & 24 & NA \\
\hline
\end{tabular}

Subjects are identified by numbers $\left(P_{n}\right)$, sex and age (in years). Diagnosis is listed as the level of the spinal lesion (traumatic) except for $P_{3}$ who was diagnosed as non-familial progressive spastic paresis $(S P)$. Duration of injury is listed in years. The functional status at the beginning of the study was indicated for each patient. Elicited clonus is reported as sustained $(S)$ or as $\leqslant 2$ beats of unsustained clonus. Subject $P_{3}$ participated in the long term phase of the study only. Clonus could not be elicited in subject $P_{8}(-)$. Dosage of cyproheptadine in both the short term (double blind crossover) trial and long term (open) trial is given in $\mathrm{mg} /$ day.

$\mathrm{NA}=$ not applicable.

study. One other subject $\left(\mathbf{P}_{5}\right)$ withdrew from the study. Each subject received, in random order, either cyproheptadine or placebo for three weeks (experimental period 1). After a washout period of one week, a three week alternative treatment began (experimental period 2). Each experimental period consisted of one week with $2 \mathrm{mg}$, three times per day, one week with $4 \mathrm{mg}$ three times daily, and the final week at $8 \mathrm{mg}$ three times daily. Cyproheptadine and placebo were supplied as oral tablets with identical appearance.

All subjects except for $P_{1}$ and $P_{2}$ received an initial training session to become familiar with the treadmill and experimental procedures. The session consisted of three or more trials, each five minutes in duration. Formal evaluation of locomotor function was performed: (1) one week after the orientation session, (2) after experimental period 1, (3) following the washout period, and (4) after experimental period 2.

Four of the subjects $\left(\mathbf{P}_{2}, \mathbf{P}_{3}, \mathbf{P}_{6}, \mathbf{P}_{7}\right)$ also participated in an open trial (long term study) in which the same assessments were performed after cyproheptadine had been administered for a minimum of six months at the optimal dose. All subjects continued to be seen in their regular therapy programmes for the duration of their participation, and anti-spasmodic medication was maintained with other medications at the same dose for at least three months before the study.

\section{Locomotor evaluation}

Locomotor evaluations were performed at the Human Gait Laboratory, School of Physical and Occupational Therapy, McGill University. The laboratory has been described in detail elsewhere ${ }^{14}$ Briefly, subjects walked on a treadmill without weight support or assistance when possible, otherwise with $40 \%$ of the body weight externally supported ( $40 \%$ BWS), by an overhead harness system. The latter alternative allowed for evaluation of the severely spastic paraparetic patients who were not able to walk at full weight on the treadmill $\left(P_{1}\right.$ and $\mathbf{P}_{2}$ ). For each trial, subjects selected what they considered to be a comfortable walking speed without knowledge of the actual speed.

\section{Temporal distance}

Footswitches were placed bilaterally beneath the heel, the head of the fifth metatarsal, and the great toe. The voltage output of each footswitch was recorded on FM magnetic tape (recording speed 3.75 ips; frequency response $0-2500 \mathrm{~Hz}$ ), then digitised and stored for analysis. Interactive computer programmes were used to determine the duration of gait cycle, stance, swing, and double support, as well as the same parameters as a percentage of the cycle duration. Cycle duration was defined as the time between one foot-floor contact and the subsequent foot-floor contact of the same leg.

\section{EMG}

Surface electrodes were attached to the gluteus maximus (GM), vastus lateralis (VL), medial hamstrings (semitendinosus/semimembranosus) (MH), tibialis anterior (TA), and medial gastrocnemius (GA) of the right lower extremity after appropriate skin preparation. Following amplification (bandpass 10-1000 $\mathrm{Hz}$ ), the EMG was simultaneously recorded with the foot switch data on FM tape. Offline, the analog EMG signal was full wave rectified, then filtered (low pass cut-off at $470 \mathrm{~Hz}$ ). The EMG was then digitised at $1 \mathrm{kHz}$ and stored for computer analysis. EMG patterns (burst onset/ offset normalised to cycle duration) were averaged at each consecutive $0 \cdot 4^{\circ}{ }_{0}$ of the cycle for the cycles selected for each subject, generating the within subject ensemble average. The amplitude of the EMG signal for each muscle was then normalised to the peak of the ensemble average for that muscle for each patient to examine differences between drug administration conditions.

\section{Joint angular displacement}

Movement in the sagittal plane was videotaped at 60 fields per second using reflective markers fixed to the lateral aspect of the following anatomical landmarks of the right leg: head of the fifth metatarsal, calcaneus, ankle joint (malleolus of the fibula), knee joint, hip joint (greater trochanter of the femur), and midline of the ribcage halfway between the iliac crest and the shoulder (greater tuberosity of the 

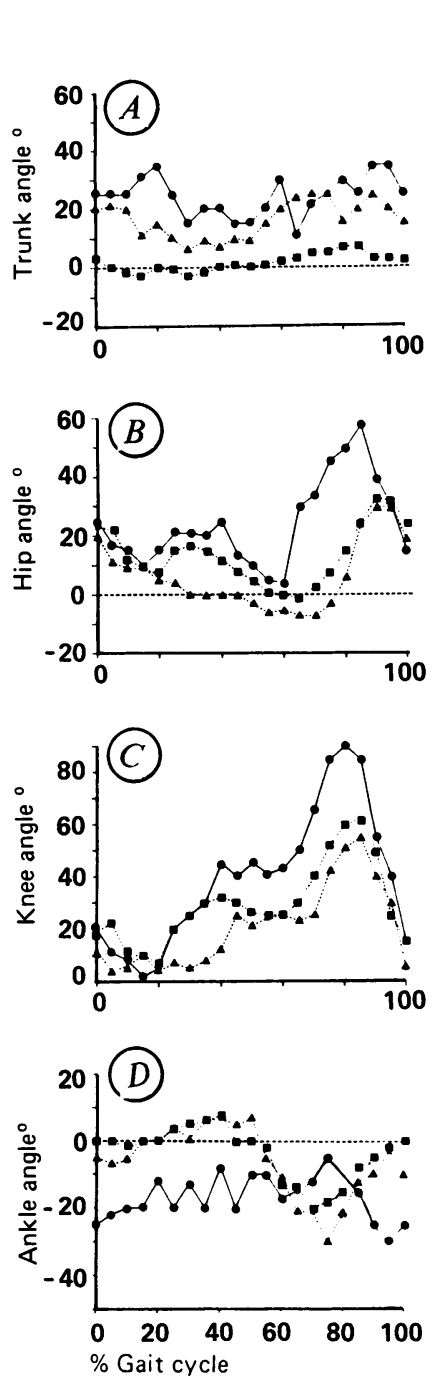

Placebo 40\% BWS

(E)

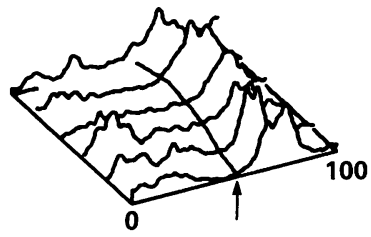

(F)

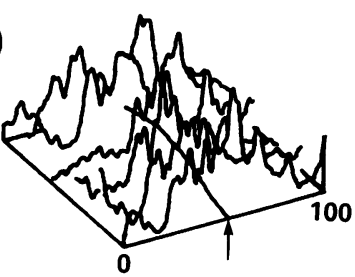

(G)

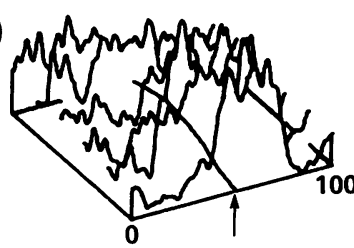

(H)

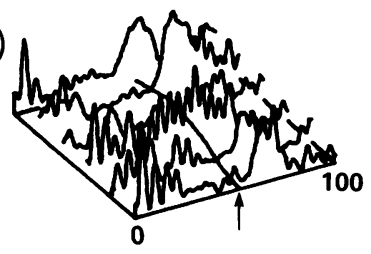

Cypro 40\% BWS

(I)

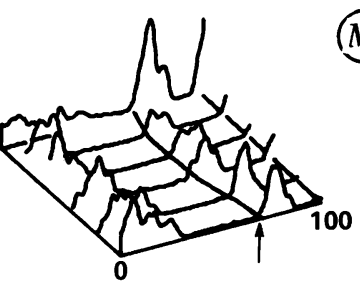

Cypro FWB

M)

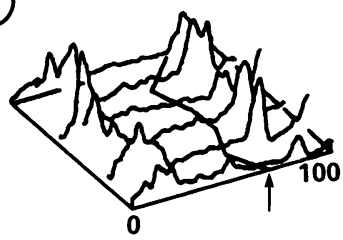

(J)
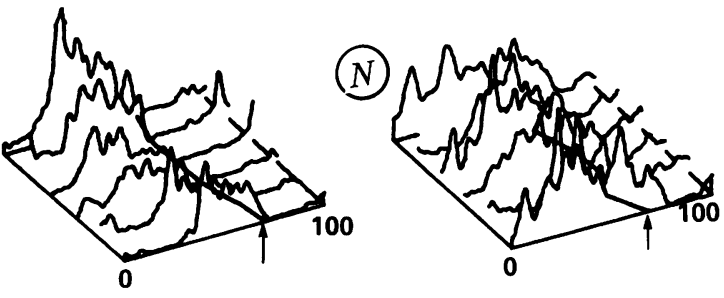

(K)

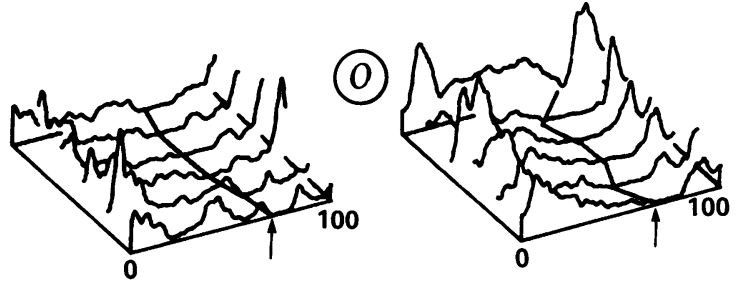

(L)
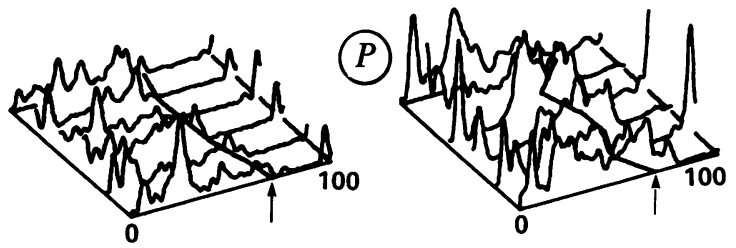

Figure 1 Kinematic and EMG pattern of a spastic paretic patient $\left(P_{2}\right)$ walking at minimum treadmill speed of 0.26 m.s.$^{-1}$ during placebo and cyproheptadine therapy. Kinematic pattern of the trunk $(A)$, hip (B), knee (C) and ankle (D) during placebo at $40 \% B W S$ (full line, full circle), long term cyproheptadine at $40 \% B W S$ (dotted line, full square), and long-term cyproheptadine at full weight bearing (dotted line, full triangle) are long term cyproheptadine at $40 \%$ pattern of the medial hamstrings ( $E, I$ and $M)$, vastus lateralis $(F, J$ and $N)$, tibialis anterior $(G, K$ and $O)$ and gisplayed at the left. The EMG pattern of the medial hamstrings $(E, I$ and $(H, L$ and $P$ ) are shown during placebo at $40 \% B W S(E-H)$, cyproheptadine at $40 \% B W S(I-L)$ and long term cyproheptadine at full weight bearing $(M-P)$. An average of six consecutive cycles is illustrated $(40 \% B W S P L A C E B O, 40 \% B W S C Y P R O$ and $F W B C Y P R O)$. These EMG data were synchronised to the normalised cycle duration defined as the period from initial foot-floor contact (0\%) to the subsequent footfloor contact $\left(100^{\circ}\right.$ ). (Same for fig 2 and 3). Arrows indicate the stance swing transition.

NB The trunk and hip angles were calculated from the vertical line, with the neutral position standing being taken as $0^{\circ}$ displacement of the trunk and hip, flexion being positive, and extension negative. Likewise, in calculating the knee and ankle angles, the neutral standing position, with the knee at full extension, and the shank axis perpendicular to the foot, was taken as $0^{\circ}$. Knee flexion and ankle dorsiflexion beyond neutral were taken as positive angular displacements, and ankle plantar flexion beyond neutral was taken as negative angular displacement.

humerus). A time code was recorded on FM tape and the videotape to permit synchronisation of EMG and angular displacement. A representative sequence of the videotape corresponding to the EMG and footswitches digitised was used to determine the pattern of sagittal displacement of the trunk, right hip, knee and ankle. The angular displacements were measured at regular intervals $(5 \%$ of step cycle) by a joint goniometer.

Additional assessments

To evaluate the severity of spasticity in each patient, EMG recordings were obtained while testing for clonus in two positions: with the knee in a position of $90^{\circ}$ flexion and with the knee in a position of maximum extension. Subjects also maintained a diary for the duration of their participation in the study, and were asked to note the frequency and different forms of involuntary movement they experienced (that is, flexor or extensor spasms or clonus).

\section{Results}

1 The effects of cyproheptadine on the walking pattern

\section{A) EMG and kinematics}

One representative patient from each group has been chosen to illustrate the effect of cyproheptadine on the EMG and kinematic pattern during locomotion.

Figure 1 shows the effect of cyproheptadine on the kinematic pattern and on the EMG of a spastic paretic subject $\left(P_{2}\right)$ who presented with frequent spasms and episodes of spontaneous, sustained clonus (see also table 1). Functionally, this subject was limited to a wheelchair for mobility. During the placebo evaluation, the 
Figure 2 Kinematic and EMG pattern of a spastic paretic patient $\left(P_{3}\right)$ walking on a treadmill at full weight bearing before (control) and during cyproheptadine therapy. Kinematic pattern of the trunk $(A)$, hip $(B)$, knee (C) and ankle (D) with (full line, full circle) and without (dotted line, full square) cyproheptadine are displayed at the left. The EMG pattern of the gluteus maximus ( $E$ and $J)$, medial hamstrings ( $F$ and $K$ ), vastus lateralis ( $G$ and $L$ ), tibialis anterior ( $H$ and $M$ ) and gastrocnemius ( $I$ and $N$ ) are shown for control ( $E-$ I) and with

cyproheptadine $(J-N)$. An average of 10 consecutive cycles is illustrated. Arrows indicate the stance swing transition.

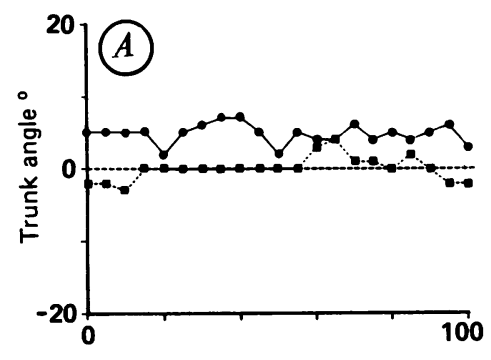

(E) Placebo
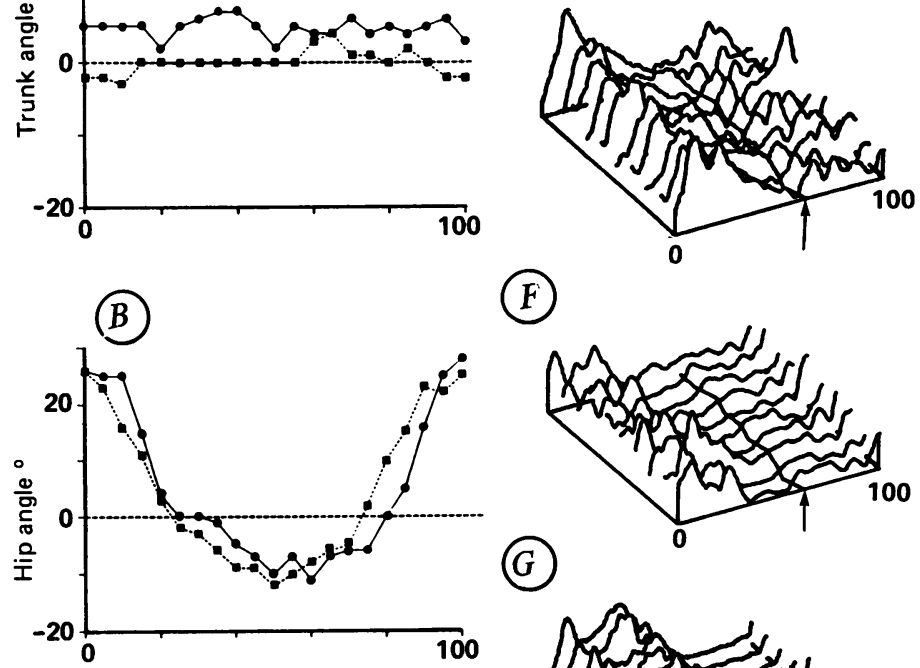

(F)

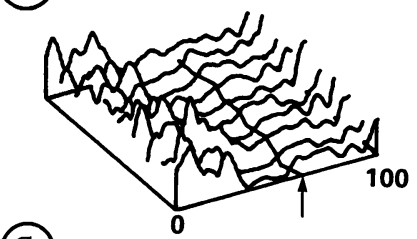

(G)
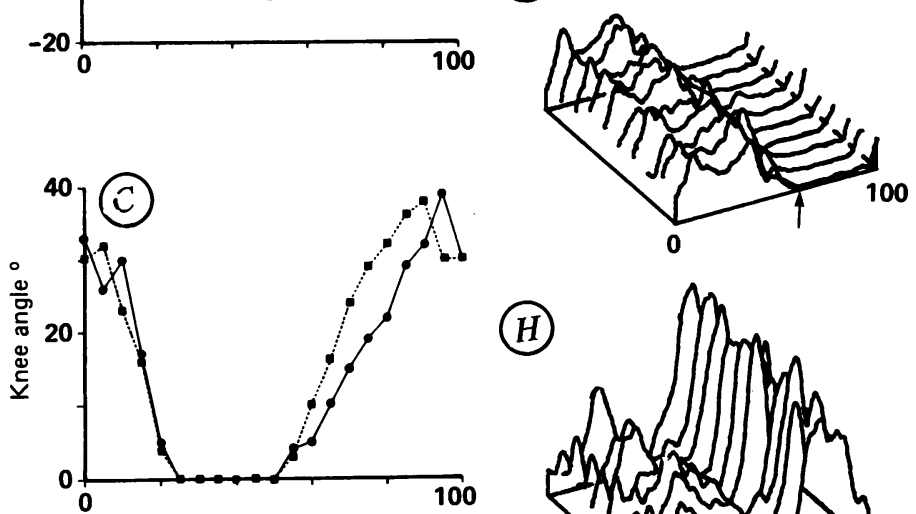

(H)
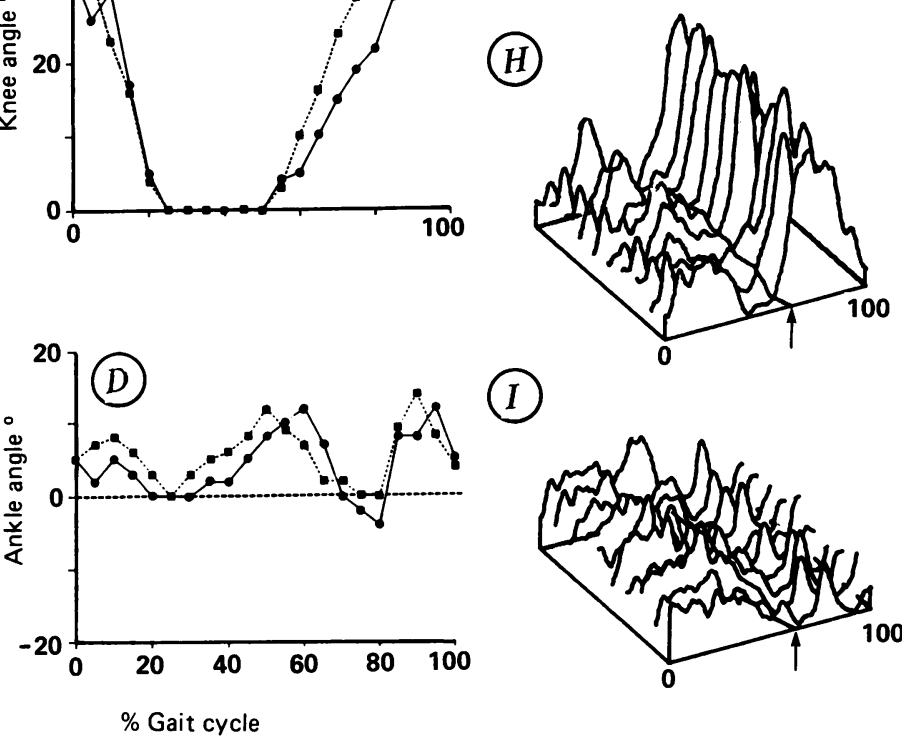

(I)

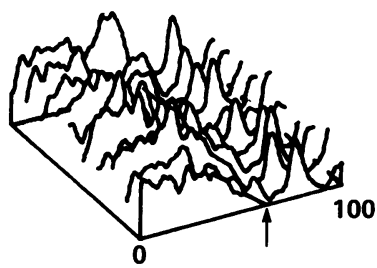

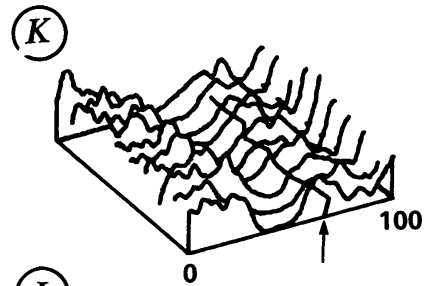

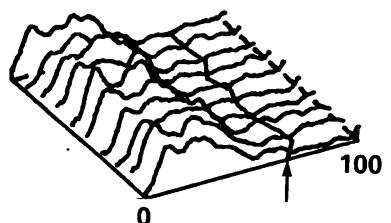

(L)

(M)
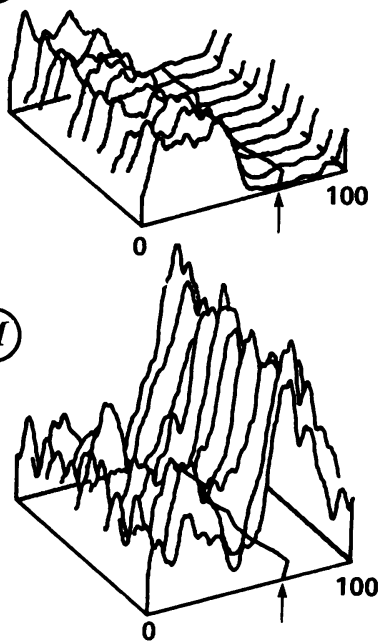

(J) Cypro

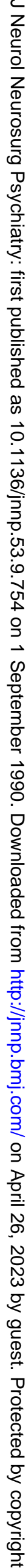

lower limb movement could not be initiated independently at full weight bearing once the treadmill was started. Thus, maximum manual assistance was required to initiate and maintain stepping. At $40 \%$ BWS, $P_{2}$ could initiate stepping in the right lower limb, if the left lower leg was manually assisted, at a minimal treadmill speed of $0.26 \mathrm{~ms}^{-1}$. The trunk, however, was inclined forward $25^{\circ}$ to $30^{\circ}$ (fig 1A). The hip collapsed into flexion repeatedly during stance. During swing, peak hip flexion was exaggerated (fig 1B). At the knee, a yield in flexion $\left(40^{\circ}-45^{\circ}\right)$ in late stance was followed by marked flexion during swing (fig 1C). The extreme flexion at both the hip and knee during swing phase appeared to be related to flexor spasms. When the patient was on placebo, the ankle remained in plantarflexion throughout the cycle ranging from $25^{\circ}$ at foot-floor contact to approximately $5^{\circ}$ plantarflexion during mid- swing (fig 1D). The presence of excessive flexion at the trunk, hip, and knee, and plantarflexion at the ankle gave rise to toe contact, instead of heel strike.

The EMG of the lower limb muscles during administration of the placebo generally showed an abnormal activation pattern (fig $1 E$ ). The medial hamstrings were characterised by two bursts of activity, consisting of a burst of small amplitude at foot-floor contact and a major burst during swing phase (fig 1E). Vastus lateralis, tibialis anterior, and gastrocnemius were characterised by prolonged activation throughout the stance and swing phases (fig 1F-H). The tibialis anterior burst occurred primarily during the stance-swing transition. Clonic discharges in gastrocnemius were commonly observed at foot-floor contact and during weight acceptance as the muscle was progressively stretched (fig $1 \mathrm{H}$ ). 


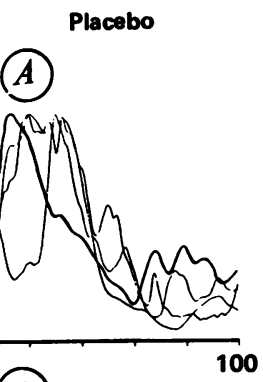

(B)

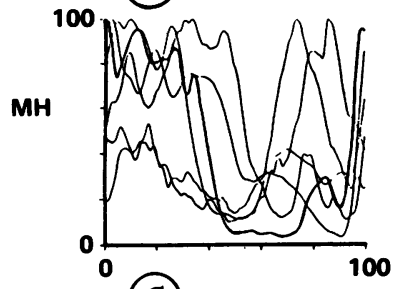

(c)

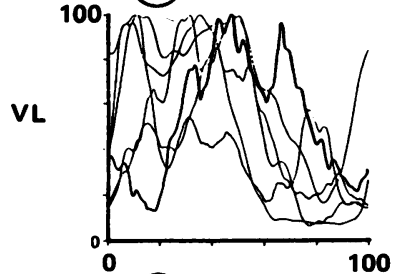

(D)

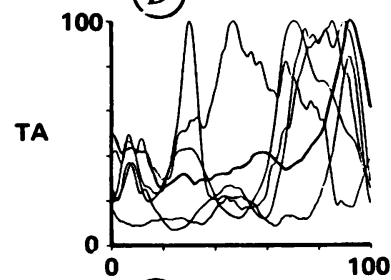

(E)

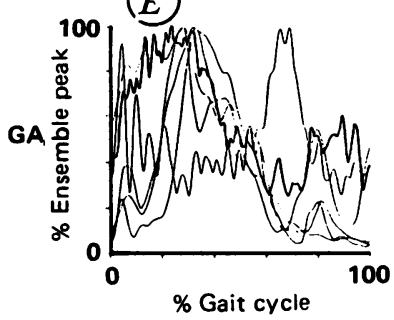

Cypro

(F)

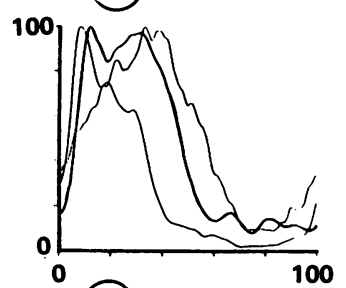

(G)

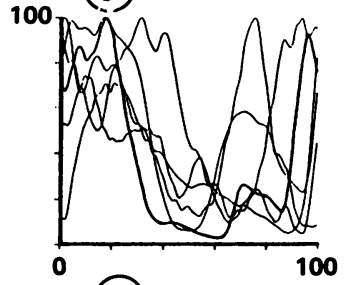

(H)

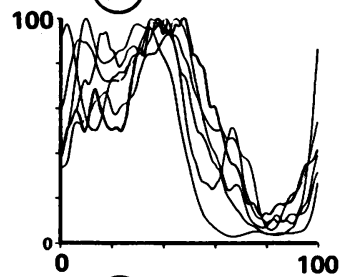

(I)

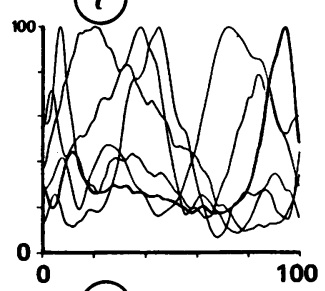

(J)

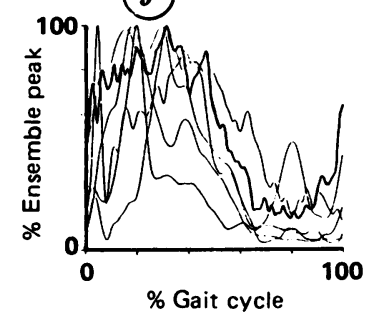

Placebo

(K)

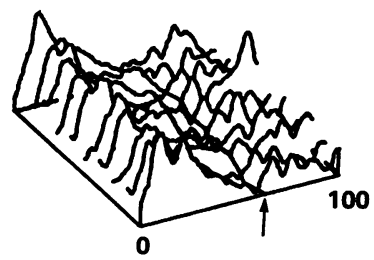

(I)

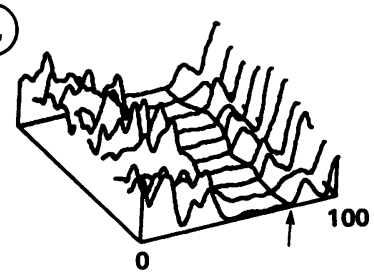

(M)

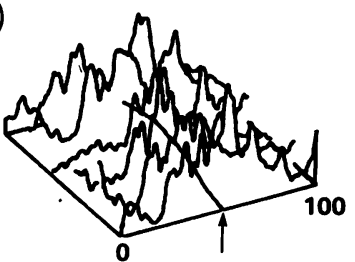

(R)

(Q)

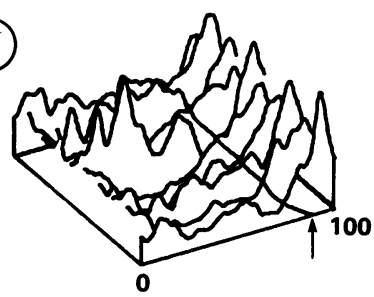

(0)

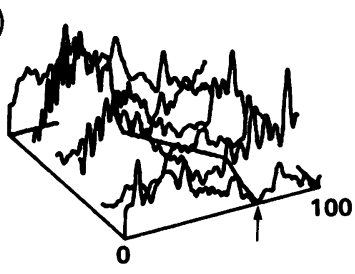

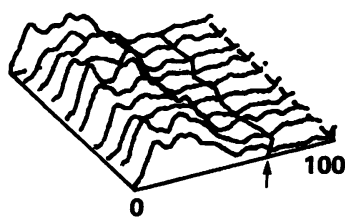
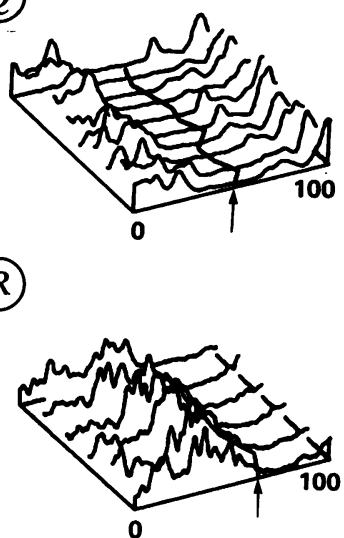

(S)

Cypro

P)

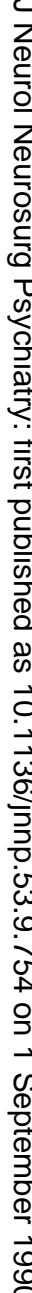

(T)
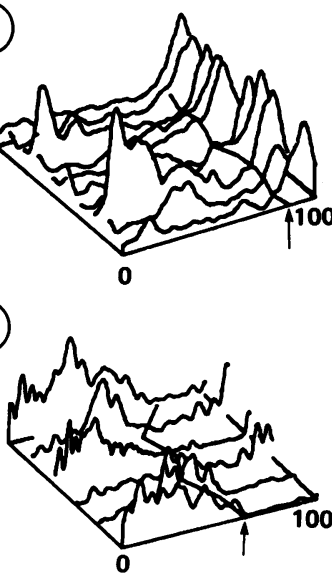

Figure 3 The EMG ensemble average for all subjects except for the gluteus maximus ( $G M$ ) ( $A$ and $F$ ) is illustrated for medial hamstrings (MH) ( $B$ and $G$ ), vastus lateralis $(V L)$ ( $C$ and $H$ ), tibialis anterior $(T A)$ ( $D$ and $I)$ and gastrocnemius $(E$ and $J)$. Shown at the right of the figure is a selected example of EMG ensemble of a selected subject for each muscle showing the effect of cyproheptadine ( $P-T)$ as compared to placebo ( $K-O)$ in different subjects for each muscle group. Figures $A$ to $T$ represent an average between six and 15 cycles. Arrows indicate the stance swing transition.

During cyproheptadine therapy ( $24 \mathrm{mg} /$ day, long term phase), a marked decrease in forward trunk flexion (fig 1A) was observed at the same treadmill speed and at $40 \%$ BWS (fig 1B). The pattern of knee movement was similar though there was less yield into flexion during stance, and a decrease in peak flexion from $90^{\circ}$ to $60^{\circ}$ during swing (fig 1C). As well, ankle angular excursion was characterised by decreased plantarflexion at foot-floor contact (fig 1D). The ankle progressively moved into dorsiflexion $\left(8^{\circ}\right)$ during stance followed by plantarflexion in late stance. The ankle then dorsiflexed during swing to clear the foot and to prepare for the next foot-floor contact. During full weight bearing with cyproheptadine, the kinematic patterns of the hip, knee, and ankle were generally similar to those patterns seen at $40 \%$ BWS with cyproheptadine. Forward flexion of the trunk during full weight bearing was greater than that observed during administration of cyproheptadine at $40 \%$ BWS and less than that observed during administration of the placebo at $40 \%$ BWS. Generally there was less hip flexion $\left(-10^{\circ}\right)$ during late stance (fig 1B), as well as a marked decrease of the hip and knee yield during midstance. The pattern at the ankle was similar to that observed with $40 \%$ BWS.

The medial hamstrings continued to produce two distinct bursts during cyproheptadine therapy (fig $1 \mathrm{I}-40 \%$ BWS). The first EMG burst began just before heel contact while the second burst occurred with eccentric 
knee extension during the swing phase. Vastus lateralis activity was restricted to the stance phase during $40 \%$ BWS (fig $1 \mathrm{~J}$ ) with relative silence throughout the swing phase. The improved coordination between medial hamstrings and vastus lateralis may be related to the greater extension at the hip (fig 1B) and at the knee (fig 1C). During cyproheptadine therapy, tibialis anterior discharged in two bursts: one at foot-floor contact and one during swing. This was related to a dorsiflexion during mid-swing for foot clearance from the treadmill belt. A marked decrease in clonus was noted in GA (fig 1L) with bursts of activities which were restricted to the stance phase. The predominantly coactivation pattern of tibialis anterior and gastrocnemius activity was replaced by alternating bursts.

At full weight bearing, the EMG pattern remained similar in many aspects to the EMG pattern described at $40 \%$ BWS except for the vastus lateralis and gastrocnemius which discharged throughout the stance until the beginning of the swing phase. This may be related to an increased load demand during weight acceptance. This marked change in EMG and kinematic pattern with cyproheptadine was also observed in the other patient $P_{1}$.

The kinematic and EMG pattern of a spastic paretic subject $\left(\mathbf{P}_{3}\right)$ from group $B$ is shown in fig 2. This subject, although he presented with spasms, could walk independently at full weight bearing on the treadmill, in contrast to the previous patient. The kinematics reveal, in the control evaluation, that the trunk was maintained in a slightly flexed position $\left(5^{\circ}\right)$ for most of the stance and the swing phase (fig 2A). At foot-floor contact, the hip was in a flexed position $\left(26^{\circ}\right)$ and passed into extension $\left(-10^{\circ}\right)$ during midstance (fig 2B). A similar flexion pattern was present at the knee at foot-floor contact with full extension of the knee from $25 \%$ to $50 \%$ of the cycle (fig $2 \mathrm{C}$ ), while the ankle dorsiflexed at foot-floor contact with a progressive plantarflexion attaining $-5^{\circ}$ at mid-swing (fig 2D).

In the control evaluation, the gluteus maximus (fig $2 \mathrm{E}$ ) showed an early EMG burst in stance with sustained EMG activation throughout swing. Medial hamstrings (fig 2F) were characterised by a main burst in early stance and remained tonically active during swing, while vastus lateralis activity was pronounced throughout the stance phase (fig $2 G$ ). Tibialis anterior had two bursts of activity, in midstance and in the swing phase (fig $2 \mathrm{H}$ ). Gastrocnemius was active throughout stance with a burst of activity in midswing (fig 2I).

With cyproheptadine, the kinematic patterns at the hip and knee were essentially unchanged, except for a slightly less plantarflexion, at the ankle. With cyproheptadine, the main burst of gluteus maximus activity was still present during the stance phase, but with a marked reduction of activity during swing (fig $2 \mathrm{~J}$ ). Vastus lateralis was active for a large portion of stance but terminated before the stance-swing transition (fig 2L). The tibialis anterior and gastrocnemius patterns remained similar (fig $2 \mathrm{M}-\mathrm{N})$.

The general effects of cyproheptadine on the EMG pattern of the lower limb muscles during walking are illustrated in fig 3. The EMG ensemble averages of the six subjects were superimposed for all the lower extremity muscles except the gluteus maximus, for which three subjects were excluded due to the presence of artefacts produced by the movement of the harness belt over the surface electrode. With placebo, the activity of the gluteus maximus muscle consisted of a main burst during stance with a lower level of EMG

Table 2

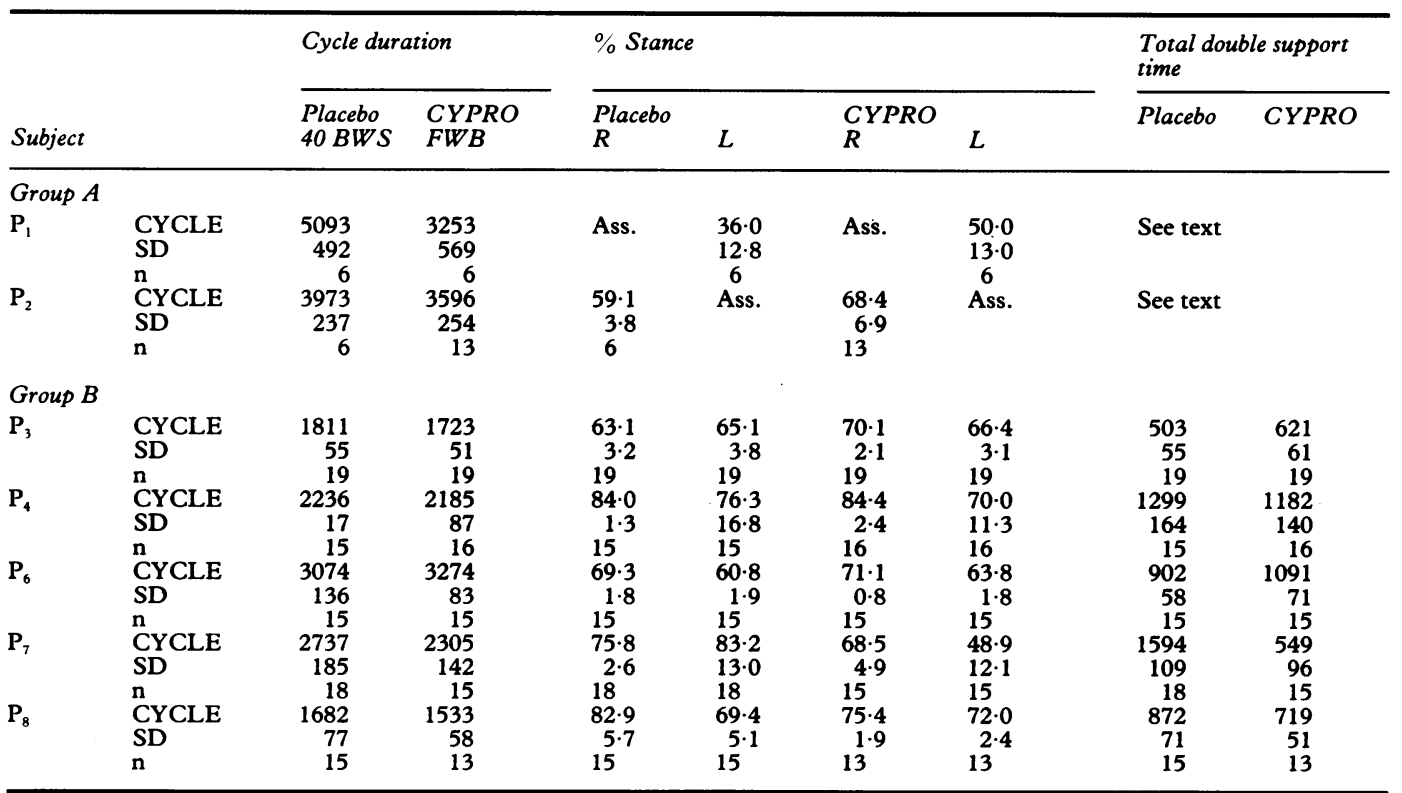

The mean cycle duration (in $\mathrm{ms}$ ), percentage stance, and total double support time (in $\mathrm{ms}$ ) are listed for all subjects in the short term study. Subjects are allocated to group A (unable to walk at full weight bearing in the control evaluation) and group B (requiring 1 or 2 elbow crutches or 1 or 2 canes to walk overground). Standard deviation (SD) and number of cycles (n) are given below. Ass = Assisted (requires maximum manual assistance) $\mathbf{R}=$ Right, $\mathrm{L}=$ Left. 
activity present during the entire swing phase (fig $3 \mathrm{~A}$ and $\mathrm{K}$ ). Medial hamstrings showed two distinct bursts of EMG activity (fig 3B). The first EMG burst was prolonged during most of the stance phase while the second small burst occurred early in the swing phase. Vastus lateralis showed a prolonged activation during the overall stance. In the example shown in fig $3 \mathrm{M}$ (also fig $3 \mathrm{C}$ ), VL was also active during the swing phase. Tibialis anterior EMG activity, shown in fig $3 \mathrm{D}$ and $3 \mathrm{~N}$, was greatest during the swing period with minimal activity at footfloor contact. For less severe patients, gastrocnemius EMG profile was characterised by an early activation at foot-floor contact or during late swing (fig $3 \mathrm{E}$ ), while in more severe patients, $P_{1}$ and $P_{2}$, clonic discharges occurred during both stance and swing (fig $3 \mathrm{E}$ and $\mathrm{O}$ ).

With cyproheptadine, the extensor burst activity was generally restricted to the stance phase with relatively silent activity during the swing phase (gluteus maximus-fig $3 \mathrm{~F}$ and $\mathrm{P}$; vastus lateralis-fig $3 \mathrm{H}$ and $\mathrm{R}$; gastrocnemius-fig $3 \mathrm{~J}$ and $\mathrm{T}$ ). The clonic discharges in gastrocnemius also decreased considerably (fig $3 \mathrm{~J}$ and $\mathrm{T}$ ). The EMG burst activity of medial hamstrings and tibialis anterior which occurred during swing did not show major changes during cyproheptadine therapy (fig 3I compared with 3D).

\section{$B$ Temporal distance}

In all subjects capable of walking at full weight bearing (group B-requiring aids to walk overground; refer to table 2), the maximum comfortable walking speed increased $(8 \%-$ $34 \%$ of control speed). Based on these observed increases in walking speed, changes in other temporal distance factors could be expected due to speed increase alone. These included a decrease in cycle duration, percentage stance and an associated decrease in the percentage of double support duration, all of which were abnormally elevated in this population (table 2). During cyproheptadine therapy, the most consistent changes involved the decrease in cycle duration ( $4 / 5$ in group B) and total double support time ( $3 / 5$ in group B). No consistent pattern was observed in the relative duration of the stance phase. In the other two subjects $\left(\mathbf{P}_{1}\right.$, $P_{2}$ ), neither of whom could walk at full weight bearing in the control or placebo evaluation (thus allocated to group A), the change in absolute duration of double support could not be determined per se, since the subjects could not move the contralateral lower limb independently during the placebo session and required maximum manual assistance. With cyproheptadine, however, the subjects could perform bilateral stepping movements independently and the phases of the gait cycle could be determined ( $\%$ total double support time: $P_{1}-38 \cdot 3 \%, P_{2}-32 \cdot 0 \%$ ).

\section{The effects of cyproheptadine on spasticity} Following administration of cyproheptadine, all subjects reported a decrease in the severity and frequency of involuntary movements. Figure 4 illustrates the change in the number of daily episodes of involuntary movements

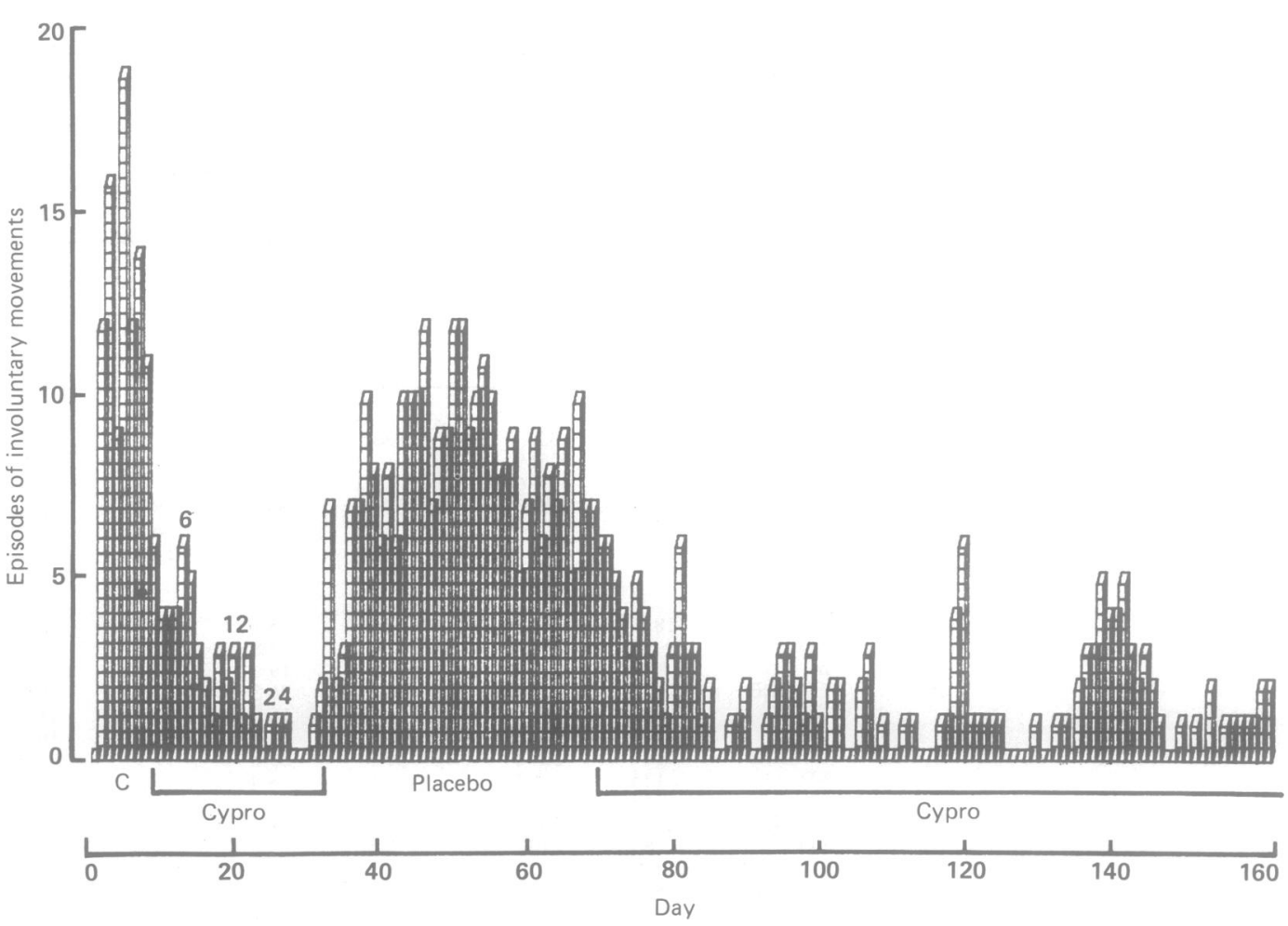

Figure 4 Figure 4 illustrates an example of the change in the number of daily episodes of involuntary movements in one subject $\left(P_{7}\right)$ over a 162 day period. Days 1-7 are the control phase, days 8-28 the short term cyproheptadine administration (see dose schedule in the Method), and days 29-32 the period of reduction of cyproheptadine. Note the progressive decrease in daily episodes of involuntary movements as the dose of cyproheptadine was increased $(6,12$ and $24 \mathrm{mg}$ (day). Following placebo administration, cyproheptadine was reinstituted on day 69 and from day 77 taken at a daily dosage of $12 \mathrm{mg} /$ day. 
reported by one subject $\left(P_{7}\right)$ over a period of 162 days. Involuntary movements were progressively abolished with a dose of six to 24 $\mathrm{mg} /$ day during the short term study. However, because two patients found their sleep disrupted while receiving this dose, it was reduced to $12 \mathrm{mg} /$ day. At this lower dose, involuntary movements were considerably reduced and no adverse side effects were present.

Ankle clonus could be elicited in six of the seven subjects (not elicitable in subject $P_{8}$ ) (see table 1). Five patients showed sustained clonus during the initial evaluation. Administration of the placebo did not alter the clonus; however, following administration of cyproheptadine, clonus was reduced from sustained manifestation to only a few beats of unsustained clonus ( $\leqslant 2$ beats) in all five subjects. In the other subject $\left(P_{2}\right)$, who showed only a few beats of unsustained clonus $(\leqslant 2)$ in the control evaluation, the clonus was unchanged by cyproheptadine.

Subjective changes reported by the subjects following cyproheptadine administration included an ability to walk longer distances, and an increase in flexibility in both the upper and lower extremities. The physiotherapists were able to place greater emphasis on gaitrelated training during administration of cyproheptadine especially in the severely spastic patients (for example: $\mathbf{P}_{2}$ : parallel bar exercises while on cyproheptadine, as opposed to only basic range of motion and stretching while on placebo). During treadmill walking, arm swing was observed more frequently and a narrower base of support was common.

Subjects generally reported drowsiness when the cyproheptadine was increased which disappeared within days at the optimal dosage. Generally subjects experienced an increase in appetite. Three subjects indicated changes in pain sensation in the back, legs and hands following administration of the placebo.

\section{Discussion \\ The effects of cyproheptadine on locomotor pattern and on manifestations of spasticity} Both groups of subjects in the present study demonstrate many abnormalities in the EMG patterns, such as disturbed timing of activity relative to the step cycle, coactivation between agonist and antagonist muscles, clonic activation of the gastrocnemius, and a generally low level of muscle recruitment (see also Fung and Barbeau 1989). ${ }^{15}$ Spastic paraparetic gait has been characterised by hyperactive spinal reflexes, impaired muscle activation patterns, a slow cadence, and poor weight bearing and balance. ${ }^{1617}$ Cyproheptadine seems to have the greatest effect on the gait pattern of the severely disabled patients in group $A$. In the two subjects who were initially unable to walk independently on the treadmill at full weight bearing $\left(P_{1}\right.$ and $\left.P_{2}\right)$, the EMG patterns following administration of cyproheptadine were modified with improved coordination between agonist and antagonist groups, and clonus was reduced. Following administration of cypro- heptadine, extensor activity was restricted to the stance phase, during which weight support was improved to the degree that the patient could now walk at full weight bearing on the treadmill.

The improved organisation in EMG activity was associated with improvements in joint angular displacement. The kinematic changes included a more erect trunk, less hip flexion during stance, a more normal excursion range at the knee and ankle, with less yielding of the knee and less plantarflexion at the ankle. The other major change was a marked reduction in the maximum hip and knee swing angles which appeared to be related to the disappearance of flexor spasms following administration of cyproheptadine. Consequently, both patients in group A could cope with more weight during treadmill walking, and retained most of the EMG and kinematic changes observed at $40 \%$ BWS. Functionally, both patients gained the ability to walk overground with walking aids. In contrast, patients in group B showed only minimal improvements in their EMG patterns and kinematics, but functional improvement was noted in that they could walk on the treadmill at higher comfortable speeds. One possible reason for this limited improvement is that the exogenous effects of 5-HT antagonist could be limited in patients who already have a more intact descending 5-HT system. However, this needs to be further elucidated.

Neurological patients have difficulty supporting their body weight while moving forward during walking. ${ }^{18}$ Abnormal weight distribution and weight transference during stance, especially at slow walking speeds, is characteristic of subjects with neurological lesions. ${ }^{1920}$ Many authors have identified increased walking speed as an indicator of functional improvement. ${ }^{20-23}$ Our results show that the maximal comfortable walking speed increased during cyproheptadine therapy and was associated with a decrease in both cycle duration and double support duration. A decrease in the duration of double support is clinically significant because it indicates that the subject is more capable of supporting his body weight on one leg. This may also indicate that there is an improvement in weight transference and/or dynamic standing balance as a result of cyproheptadine therapy. Functional improvement was further evidenced in that patients previously requiring BWS were able to walk bearing their full weight during cyproheptadine therapy.

Employing the BWS approach essentially alters the load borne through the lower extremities and possibly also decreases the stretch imposed upon muscles. Preliminary results indicate that providing BWS permits the expression of a more normal locomotor pattern as measured by EMG and kinematic changes. ${ }^{24}$ The combination of medication with BWS has provided promising preliminary results. 25

In this study, cyproheptadine also considerably decreased the duration of ankle clonus observed in all the patients who previously presented this manifestation of spasticity. A 
dose-dependent action of cyproheptadine on clonus and spasms has also been reported. ${ }^{12}$ All the spinal cord injured patients were stabilised on other anti-spasmodic medications before participating in this study. The further decrease in clonus and spasms in patients who were already on baclofen suggests that cyproheptadine is a relatively powerful antispasmodic agent and may work complementarily with other anti-spasmodic medications. To strike a balance between optimising the antispasmodic effects of the drug and minimising the adverse side effects, monitoring the individual dose is necessary to determine the required amounts. As such, a lower daily dose of cyproheptadine was selected for subject $P_{7}$ in the long term aspect of the study. It is important to note that there were also changes in other physiological and behavioural functions known to be associated with the serotonergic system, such as pain and sensory perception, sleep and level of arousal, and bladder and sexual function. This, however, needs to be further explored.

Possible mechanisms underlying the action of cyproheptadine

The general modification of the EMG patterns may be related to the 5-HT blocking action of cyproheptadine as shown in the spinal animal. ${ }^{81011}$ Such animals provide a good model because all the descending bulbospinal serotoninergic terminals have degenerated after spinal transection. Administration of 5HT agonists in chronic spinal rats placed on a platform with the hindlimbs hanging through the holes produced a marked increase of EMG amplitude of hindlimb muscles. ${ }^{8}$ At that time some spasticity-like phenomena such as clonus and spasms were observed. Administration of cyproheptadine completely reversed these 5HT agonist-induced effects.

Recently, Rossignol et al, ${ }^{10}$ and Barbeau and Rossignol $^{11}$ studied the effects of 5-HT agonists and antagonists on the walking pattern and reflexes in chronic spinal cats. Administration of 5-HT agonists considerably increased EMG burst amplitude of extensor and flexor muscles. Moreover, the flexion reflex response to stimulation delivered to the dorsum of the paw through implanted wires was considerably increased following serotonergic agonist injection; the response was reversed following cyproheptadine injection. The increase in cutaneous excitability combined with an increase in motor neuron excitability by $5-\mathrm{HT}$ may explain the spasticity-like behaviours such as clonus and spasms which were frequently observed during walking. ${ }^{11}$ This 5-HT effect agrees with previous studies indicating that 5HT reduces the threshold for rhythmic firing of alpha motor neurons induced by a depolarising current pulse. ${ }^{2627}$ An alternative explanation proposed by Shibuya and Anderson," Barbeau and Bedard, ${ }^{28}$ and Nygren et $a l^{29}$ is that 5-HT produces a greater effect in chronic spinal animals because of the development of denervation supersensitivity of 5-HT receptors below the transection. Cyproheptadine has been postulated to change neuronal excitability by blocking denervation supersensitive postsynaptic 5-HT receptors. ${ }^{8}$ The modification of the EMG pattern during locomotion in spastic paraparetic patients, as well as the decrease in clonus and spasms, could be related to similar modulatory actions by cyproheptadine. This relationship requires further investigation.

The current results suggest that cyproheptadine may function as an anti-spasmodic medication that enhances locomotor function in spastic paretic patients. The relationship between the anti-spasmodic action of cyproheptadine, and its effects on the modulation of the locomotor pattern, need to be further investigated. It is also necessary to continue with randomised control drug trials to determine which patient populations may preferentially benefit from cyproheptadine therapy, and to discover the underlying mechanisms responsible for these effects.

This work was supported by the Medical Research Council of Canada. Medication was provided as part of an operating grant by Merck Sharp and Dohme. H Barbeau is a Research Scholar of the "Fonds de la Recherche en Santé du Québec". We thank Dr the "Fonds de la Recherche en Sante du Quebec". We thank Dr S Gauthier for performing the neurological evaluations, $M$
Visintin, L Salvo, J Stewart and J Gordon for their technical assistance, and E Riley and the Montreal Rehabilitation and Dr N Bradley for their helpful comments on the manuscript.

1 Hammond DL. Control systems for nociceptive afferent processing. The descending inhibitory pathways. In: Yaksh TL, ed. Spinal Afferent Processing. New York: Plenum Press 1986:363-90.

2 Aghajanian GK, Vandermaelen CP. Specific systems of the reticular core: serotonin. In: Mountcastle VB, Bloom FE, Geiger SR, eds. The nervous system Vol 4. Intrinsic regulatory systems of the brain. Bethesda: Amer Physiol Soc regulatory system

3 Myslinski NR, Anderson EG. The effect of - serotonin precursors on alpha and gamma motoneuron activity. $J$ Pharmacol Exp Ther 1978;204:19-26.

4 White GR, Newman RS. Facilitation of spinal motoneurons excitability by 5 -hydroxytryptamine and noradrenaline. Brain Res 1980;188:119-27.

5 Dalhström A, Fuxe $K$. Evidence for the existence of monoamine neurons in the central nervous system. II. Experimentally induced changes in the intraneural amine levels of bulbospinal neuron systems. Acta Physiol Scand 1965;64:1-85.

6 Segu L, Calas A. The topographical distribution of serotonergic terminals in the spinal cord of the cat: quantitative radioautographic studies. Brain Res 1978;153:449-64.

7 Barbeau H, Bédard P. La sérotonine et la spasticité. Physioth Can 1980;32:88-92.

8 Barbeau H, Filion $M$, Bedard P. Effects of agonists and antagonists of serotonin on spontaneous hindlimb EMG activity in chronic spinal rats. Neuropharmacol 1981;20:99-107.

9 Shibuya T, Anderson EG. The influence of chronic cord transection on the effects of 5-hydroxytryptophan, Ltryptophan and pargyline on spinal neuronal activity. $J$ Pharmacol Exp Ther 1968;164:185-90.

10 Rossignol S, Barbeau H, Julien C. Locomotion of the adult chronic spinal cat and its modification by monoaminergic agonists and antagonists. In: Goldberger $M$, Gorio $A$, agonists and antagonists. In: Goldberger $M$, Gorio A, malian Spinal Cord. Padova: Fidia Research Series Vol 3, malian Spinal Cord. Padova:

11 Barbeau $\mathrm{H}$, Rossignol S. The effects of serotonergic drugs on the locomotor pattern and on the cutaneous reflexes of the the locomotor pattern and on the cutaneous reflexes

12 Barbeau H, Richards CL, Bédard P. Action of cyproheptadine in spastic parparetic patients. $J$ Neurol heptadine in spastic parparetic pati

13 Wainberg M, Barbeau H, Gauthier S. Quantitative assessment of the effect of cyproheptadine on spastic paretic gait: A preliminary study. J Neurol 1986;233:311-4.

14 Barbeau H, Wainberg M, Finch L. Description and application of a system for locomotion rehabilitation. Med Biol Engng Comput 1987;25:641-4.

15 Fung J, Barbeau H. A dynamic EMG profile index to quantify muscular activation disorder in spastic paretic gait. Electroenceph Clin Neurology 1989;73:233-44.

16 Conrad B, Benecke R, Meinck AM. Gait disturbance in para-spastic patients. In: Delwaide PS, Young RR, eds. para-spastic patients. In. Delwaide PS, Young RR, eds. Elsevier, 1985:155-74.

17 Barbeau H, Fung J, Stewart J, Visintin M. Impairment of 
spastic paraparetic gait: Implications for new rehabilitation strategies. Proc of Can Soc for Biomech 1988;5:12-16.

18 Chin PL, Rosie A, Irving M, Smith R. Studies in hemiplegic gait. In: Rose FC, ed. Advances in Stroke Therapy. New York: Raven Press, 1982:197-211.

19 Carlsoo S, Dalhof A, Holm J. Kinetic analysis of the gait in patients with hemiparesis and in patients with intermittent claudication. Scand J Rehabil Med 1974;6:166-79.

20 Mizrahi J, Susak Z, Heller L, Najenson T. Objective expression of gait improvement during rehabilitation by time distance parameters of the stride. Med Biol Engng Comput 1982a;20:628-34.

21 Brandstater M, de Bruin H, Gowland C, Clark B. Hemiplegic gait: Analysis of temporal variables. Arch Phys Med plegic gait: Analysis of

22 Holden MK, Gill RM, Magliozzi MR, Nathan J, PiehlBaker L. Clinical gait assessment in the neurologically impaired. Reliability and meaningfulness. Phys Ther 1984;65:35-40.

23 Mizrahi J, Braun Z, Najenson T, Graupi D. Quantitative weight bearing and gait evaluation of paraplegics using functional electrical stimulation. Med Biol Eng Comput 1985;23:101-7.

24 Visintin M, Barbeau H. The effects of body weight support on the locomotor pattern of spastic paretic patients. Can J Neurol Sci 1989;16:315-25.

25 Fung J Stewart J, Barbeau $\mathrm{H}$. The combined effect of clonidine and cyproheptadine with interactive locomotor clonidine and cyproheptadine with interactive locomotor training on

26 Hounsgaard $\mathrm{J}$, Hultborn $\mathrm{H}$, Kiehn $\mathrm{O}$. Transmittercontrolled properties of alpha-motoneurones causing long-lasting motor discharge to brief excitatory inputs. In Freund HJ, Buttner U, Cohen B, Noth J, eds. The Oculomotor and Skeletal Motor Systems: Differences and Similarities. Progress in brain research Vol 64. Amsterdam: Elsevier, 1986:39-49.

27 Hounsgaard J, Kiehn O. $\mathrm{Ca}++$ dependent bi-stability induced by serotonin in spinal motoneurons. Exp Brain Res $1985 ; 57: 422-5$.

28 Barbeau H, Bédard P. Denervation supersensitivity to $5-$ hydroxytryptophan in rats following spinal transection hydroxytryptophan in rats following spinal transection 1981;20:611-16.

29 Nygren L, Fuxe K, Jonsson G, Olson L. Functional regeneration of 5-hydroxytryptamine nerve terminals in the rat spinal cord following 5-6 dihydroxytryptamine induced degeneration. Brain Res 1974;78:377-94.
Osler on the aetiology of tabes dorsalis (Locomotor Ataxia)

Many neurologists now have little experience of tabes dorsalis but we all assume its syphilitic aetiology. It is of interest to reflect on opinons when the disease was rife.

In his fourth edition, Osler ${ }^{1}$ recalls that of a series of 8642 cases in the neurological dispensary of Johns Hopkins, 89 were locomotor ataxia; males outnumbered females 10:1, and it was a rarity in the black population. The role of syphilis was not a constant factor. According to Erb, Fournier and Gowers, from $50-75^{\circ}$ o of all cases there is a history of this disease. Erb's recent figures are most striking; of 300 cases of tabes in private practice $89^{\circ}$ o had had syphilis. Moebius goes so far as to say, "The longer I reflect upon it the more firmly I believe that tabes never originates without syphilis."

Osler mentions "excessive fatigue, overexertion, injury, exposure to cold and wet, and sexual excesses are all assigned causes . . . alcohol excess does not seem to predispose to this disease . . . there are now a good many cases on record of the existence . . . in both husband and wife."

One fascinating clinical titbit: "when optic atrophy develops early and leads to blindness, ataxia rarely if ever supervenes ... a a sort of antagonism between the ocular symptoms and the progress of the ataxia. Charcot laid considerable stress upon this, and both Dejerine and Spiller have since emphasized the point."

JMS PEARCE

1 Osler W. The principles and practice of medicine, 4th ed Edinburgh and London: Young J Pentland, 1901:920-8. 